\title{
The Implications of Simultaneous Smoking Initiation for Inferences about the Genetics of Smoking Behavior from Twin Data
}

\author{
Michele L. Pergadia, ${ }^{1,3}$ Andrew C. Heath, ${ }^{1}$ Arpana Agrawal, ${ }^{1}$ Kathleen K. Bucholz, ${ }^{1}$ Nicholas \\ G. Martin, ${ }^{2}$ and Pamela A. F. Madden ${ }^{1}$
}

Received 29 Apr. 2005-Final 22 Dec. 2005

We examined early social influences across stages of smoking within the context of a twin study using an environmental exposure specific to smoking: whether twins started smoking at the same time ("simultaneous smoking initiation": SSI). We expected that SSI would be a good index of shared social influences on smoking initiation. Rates of SSI were indeed significantly higher in MZ twins and in twins who shared peers and classes, as well as in male twins. With the exception of regular smoking in females, we found no significant difference in estimates of genetic and environmental parameters between SSI and non-SSI pairs for any of the smoking measures that we examined (DSM-IV and Fagerstrom HSI measures of nicotine dependence; DSM-IV nicotine withdrawal; heavy smoking; and in males, regular smoking). For regular smoking in females, allowing for additional shared environmental influences associated with SSI only modestly reduced our estimates of additive genetic variance ( $56 \%$ vs. $68 \%$ ). These results indicate the important social influences that may occur for smoking initiation do not appear to seriously bias estimates of genetic effects on later stages of smoking.

KEY WORDS: Environmental influences; genetic influences; simultaneous smoking initiation; twin data.

\section{INTRODUCTION}

Approximately $90 \%$ of Australian young adults (e.g., Lessov et al., 2004), $80 \%$ of US young adults (Maes et al., 2004) and $60 \%$ of US adolescents (e.g., CDC, 2004) have tried cigarettes at some point in their lives. While experimenting with cigarettes is clearly a common phenomenon, there is variability in the factors surrounding this event, such as initial reactions to the first cigarette (Pomerleau et al., 1993),

\footnotetext{
${ }^{1}$ Department of Psychiatry, Washington University School of Medicine, St. Louis, MO, USA.

${ }^{2}$ Queensland Institute of Medical Research, Brisbane, Australia.

3 To whom correspondence should be addressed at Department of Psychiatry, Washington University School of Medicine, 660 S. Euclid Avenue, Campus Box 8134, St. Louis, MO, 63110, USA. Tel.: + 1-314-286-2270. Fax: +1-314-286-2213. e-mail: michelep@matlock.wustl.edu
}

peer, sibling and parental use (Vink et al., 2003), parental monitoring (Simons-Morton, 2002), local cigarette taxation rates and tobacco industry marketing (Thomson et al., 2004), in addition to possible differences in genetic vulnerability (Heath et al., 1993; Li et al., 2003; Madden et al., 2004; Maes et al., 2004). To complicate the matter further, an individual's genetic background may be correlated with the environmental factors that foster not only risk to try cigarettes but the associated factors that predict continued use (Cleveland et al., 2005). Some $36 \%$ of adolescents who try cigarettes progress to daily smoking (CDC, 1998). Over $70 \%$ of adolescent daily smokers report that they have tried to quit, but over $85 \%$ persist in smoking (CDC, 1998). Persisting in the smoking habit significantly increases risk for tobacco-related lung cancer (Peto et al., 2000), and remains the single greatest preventable cause of death 
(CDC, 2005), taking more that 400,000 lives in the US every year. US adult male and female smokers lose an average of 13 and 15 years of life, respectively, as a direct result of cigarette use (CDC, 2002).

It has been suggested that prevention efforts targeting social influences on smoking might prove to be more beneficial from a public health perspective than identifying individual genetic risk for smoking behavior (Merikangas and Risch, 2003). This argument is defensible if early social influences also have persistent effects on later long-term smoking outcomes; but indefensible if many of the risk-factors for later stages of smoking are unrelated or only weakly related to social influences on initiation. Preventing the emergence of a new generation of smokers may be considered desirable, but abandoning efforts to develop better methods to help existing smokers to quit is surely undesirable. Important genetic influences have been reported for initiation of smoking (Heath et al., 1993; Li et al., 2003; Madden et al., 2004; Maes et al., 2004), persistent smoking (Heath and Madden, 1995; Madden et al., 1999, 2004), quantity smoked (Koopmans et al., 1999), and nicotine dependence (Kendler et al., 1999; Lessov et al., 2004; Maes et al., 2004) based on studies of many different twin populations. However, given the importance of early social influences on initiation of smoking (Kendler and Gardner, 1998; Madden et al., 2004; Slomkowski et al., 2005), the validity of inferences about genetic influences on smoking based on results from twin studies might be questioned, given that one unique aspect of twin pairs, growing up with a same age sibling, might make social influences especially important in this group, and perhaps contribute differentially to the resemblance of monozygotic versus dizygotic pairs.

Our aim in this paper is to take advantage of this unique social situation of twin pairs to investigate the importance of early environmental exposures specific to smoking for later smoking outcomes. Specifically, we investigate smoking outcomes in twins who start smoking at the same time, which we term "simultaneous smoking initiation (SSI)", compared to other twin pairs. To the extent that early social factors are having important lasting effects on later stages of smoking, we would expect to find that SSI twin pairs would show much higher concordance for later stages of smoking than other twin pairs. A second aim was to explore the factors associated with SSI (e.g., shared peers), and the degree to which these might mediate SSIs influence on smoking outcomes.

\section{METHOD}

\section{Participants}

Participants were male and female twins born 1964-1971 from the Australian Twin Registry ("1989 Australian Adult Twin Cohort"), who were first surveyed by mailed questionnaire in 1989 and later interviewed between 1996 and 2000. These twins were volunteered by their parents, in response to media appeals and appeals through the Australian school system, in 1980-1982 (see Heath et al., 2001; Knopik et al., 2004 for further details of sample). Participants were interviewed by telephone using a structured diagnostic assessment for DSM-IV (APA, 1994) nicotine dependence, alcohol dependence, major depression, panic disorder, and childhood conduct disorder, as well as non-diagnostic assessments of social anxiety and suicidality, a screen for bipolar disorder, and a detailed history of consumption of alcohol, cigarettes and other forms of tobacco, and illicit drugs. Twins were also queried as to whether they started to smoke at the same time, the degree to which they shared peers from ages 6-13, the extent to which they shared the same classes in primary school and high school (age 12 and older in Australia), and the extent to which they dressed alike between the ages of 6-13 (in same-sex twins). The interview was a modified version of the Semi-Structured Assessment on the Genetics of Alcoholism (SSAGA; Bucholz et al., 1994) and the smoking section was modified from the Composite International Diagnostic Interview (CIDI; Cottler et al., 1991). Telephone interview data were available from a total of 6257 individual twins (3454 women and 2803 men) including both twins from 1192 monozygotic (MZ) pairs (698 female pairs), 908 same-sex dizygotic (DZ) twin pairs (513 female pairs) and 661 unlike-sex pairs. Data were also collected from 735 single twins. At the time of interview, all respondents ranged in age from 24- to 36year-old (mean age $=30$ ). For the purposes of the present paper, most analyses were limited to individuals who had ever used cigarettes (3028 women and $2554 \mathrm{men}$ ), and twin pairs where both twins had used cigarettes (558 MZF, 419 MZM, 424 DZF, 333 DZM, and 559 DZ unlike sex pairs).

\section{Measures}

Simultaneous Smoking Initiation (SSI)

Participants who endorsed both themselves and their twin as "trying" cigarettes were asked further: 
"Who tried first, you or your twin or was it both at the same time?" Starting to smoke at the same time, or simultaneous initiation (SSI) was coded as yes (or ' 1 ') if participants reported that they started at the same time or as ' 0 ' if participants reported that they started to smoke separately. If twins reported that they did not know whether they started at the same time, they were coded as missing for this variable $(N=542)$, and are excluded from all analyses. For genetic analyses at the twin pair level, twins were classified as an SSI pair, if both twins reported SSI, and were classified as a non-SSI pair if either twin reported that they started separately.

\section{Regular Smoking}

Participants who endorsed "trying" cigarettes were asked to further describe their lifetime smoking behavior as: "(1) I have only smoked one or two times 'just to try', (2) I have only smoked occasionally, never as often as one day a week for three weeks or more, (3) I have smoked as often as one or two days a week (but never more than one or two days a week) for a period of three weeks or more, or (4) I have smoked daily, or nearly every day, for a period of three weeks or more." Next they were asked: "How many times in your life have you smoked a cigarette?: (A) only once or twice in my entire life; (B) 20 times or less in my entire life, but more than twice; (C) fewer than 100 times in my entire life, more than 20 times; (D) 100 or more times." Participants were coded as regular smokers (or '1') if they endorsed smoking at least weekly or daily and 100 or more cigarettes lifetime, and were coded as ' 0 ' if they had experimented with cigarettes but never as much as weekly or fewer than 100 cigarettes lifetime. Among individuals who smoked at least 100 or more cigarettes lifetime, only 30 did not smoke at least weekly or daily, and only 160 who smoked at least weekly never smoked at least 100 cigarettes lifetime, these two groups being coded as zero for regular smoking.

\section{Heavy Smoking}

Regular smokers as defined above, were asked in reference to the period of time when they smoked the most, "how many cigarettes did you smoke on a typical day, on those days when you smoked" from $1-5,6-10,11-15,16-19,20-25,26-39$, to 40 or more. Heavy smoking was coded as ' 1 ' if the individual was a regular smoker and reported smoking 20 or more cigarettes on a typical day, regular smokers were coded as ' 0 ' for heavy smoking if they never smoked 20 cigarettes per day during the time when they were smoking the most.

\section{DSM-IV Nicotine Dependence}

Regular smokers who met criteria for DSM-IV nicotine dependence, which included the clustering of three or more symptoms within a 12-month period, were coded as ' 1 ' for nicotine dependence, whereas regular smokers who did not meet criteria for DSMIV nicotine dependence were coded as ' 0 '.

\section{DSM-IV Nicotine Withdrawal}

Regular smokers were asked "about problems [they] might have had in the first $24 \mathrm{~h}$ after [they] stopped or cut down or when [they] were unable to smoke". Individuals met criteria for DSM-IV nicotine withdrawal (coded as ' 1 '), if they endorsed 4 or more of the symptoms or if the individual endorsed smoking cigarettes to relieve or avoid withdrawal symptoms.

\section{Heaviness of Smoking Index (HSI)}

Following the definition for (HSI) provided by Heatherton et al. (1989, 1991), which sums across: (i) number of cigarettes smoked per day (on a scale from 0 to 3 ) and (ii) time to first cigarettes in the morning (on a scale from 0 to 3 ), regular smokers (defined above) reporting typical daily consumption in the range of 1-10 cigarettes per day (cpd) were coded as ' 0 ', 11-25 cpd as ' 1 ', 26-39 cpd as ' 2 ', and 40 or more cpd as ' 3 '. In reference to the same period participants were asked "how soon after you woke up did you smoke your first cigarette?" Individuals who reported over 1 -h were coded as ' 0 ', 31-60 min as ' 1 ', 6-30 $\mathrm{min}$ as ' 2 ', and responses within the first $5 \mathrm{~min}$ as ' 3 '. For the final binary measure of HSI dependence used in these analyses, scores greater than 3 were coded as ' 1 ', and scores from 0 to 3 were coded as ' 0 '.

\section{Shared Peers/Shared Same Classes/Dress Alike}

For further characterization of SSI, we examined the associations with three potentially influential environmental measures: (1) individuals were code as ' 1 ' for sharing peers if they reported that between the ages of 6-13 they usually or always shared peers with their cotwin, and ' 0 ' if they reported sharing friends 
sometimes, rarely or never; (2) as ' 1 ' if they reported that they were usually or always in the same classes as their cotwin in high school, and ' 0 ' if they reported sometimes, rarely or never; and (3) same-sex twins were scored as ' 1 ' if they reported that between the ages of 6-13 they usually or always dressed alike, and ' 0 ' if they reported that they dressed alike sometimes, rarely or never. For analyses at the twin pair level, twins were considered concordant for these measures, if both twins endorsed the same level of similarity, and were considered discordant if either twin reported a level of less similarity.

\section{Analytical Approach}

Prevalence rates for all measures were estimated using SAS (1999). We examined what factors predict becoming a joint initiator (or SSI), in twins from pairs concordant for ever smoking, using backward selection logistic regression. Analyses were conducted in Stata (2003), using the Huber-White Robust Variance Estimator to correct for the nonindependence of measures in twins. Variables considered included: zygosity and gender (using dummy variables for zygosity with dizygotic females as the comparison group), DSM-IV major depression, DSM-IV conduct disorder, DSM-IV panic disorder, social anxiety, shared peers, sharing the same classes, and dressing alike. Logistic regression analyses also examined the extent to which any associations between SSI and smoking outcomes might be accounted for by the factors found to be associated with SSI.

Univariate genetic models (Eaves et al., 1978; Neale et al., 2002) were fitted for each categorical smoking measure by the method of maximum likelihood, using Mx (Neale et al., 2002) to estimate the proportion of the total variance in each smoking measure that could be explained by additive genetic factors (A), environmental influences shared by members of a twin pair (C), and non-shared environmental influences (E), using data from all five zygosity groups. Analysis of regular smoking was limited to pairs concordant for any cigarette use. Analyses of other smoking measures were limited to pairs concordant for regular cigarette use. Because the $\mathrm{MZ}$ twin correlation for regular smoking was less than twice that of $\mathrm{DZ}$ twins, tests for non-additive genetic influences on this measure, and other measures of smoking behavior conditioned on regular smoking, were not conducted. In analyses testing for the moderating influence of SSI on other measures of smoking behavior (regular smoking, heavy smoking, DSM-IV nicotine dependence, DSM-IV nicotine withdrawal, and HSI), we refit models after subdividing pairs into those reporting concordant SSI compared to those not reporting SSI. A significant moderation effect was noted if likelihood-ratio Chi-Square tests of heterogeneity indicated elevated familial effects on later-stage smoking outcomes in twins who started smoking at the same time compared to those who started separately. When significant heterogeneity was found, we conducted additional genetic model-fitting analyses in which additional shared environmental (or genetic) parameters were estimated for twin pairs reporting SSI, and for twin pairs reporting shared peers (the only other environmental measure that was strongly associated with SSI).

\section{RESULTS}

\section{Descriptive Statistics, Correlates of SSI}

About $89 \%$ of all respondents reported trying cigarettes at least once, and of these individuals $56.6 \%$ progressed to regular smoking, with this latter proportion lower in MZ twins $(52.9 \%)$ than in DZ twins $(58.2 \%$ : OR $=0.72,95 \%$ CI $0.58-0.90)$. Among regular smokers, $61.2 \%$ reported a history of DSMIV nicotine dependence, $43.3 \%$ DSM-IV nicotine withdrawal, and $19.3 \%$ met HSI criteria for nicotine dependence. Heavy smoking was less common in women $(33.7 \%)$ than in men $(47.8 \%$ : OR $=0.66,95 \%$ CI 0.51-0.84). Simultaneous Smoking Initiation (SSI) was reported by $37.8 \%$ of ever smokers, but probability of SSI varied significantly as a function of gender (less common in females: $\mathrm{OR}=0.59,95 \%$ $\mathrm{CI}=0.47-0.74$ ), and zygosity (more common in $\mathrm{MZ}$ twins: $\mathrm{OR}=1.54,95 \%$ CI 1.23-1.92; less common in DZ unlike-sex twins: $\mathrm{OR}=0.18,95 \%$ CI $0.13-0.24$ ). Overall rates of self-report SSI (ignoring cotwin report) in women were $53.4 \%$ of MZ twins, $36.7 \%$ of DZ same-sex twins and $20.1 \%$ of women from unlike-sex twins; with corresponding percentages in men of $60.3 \%, 49.7 \%$ and $15.1 \%$. Like SSI, sharing of peers was more commonly reported by MZ twins $(\mathrm{OR}=2.54,95 \%$ CI $1.96-3.29)$ and less commonly reported by unlike-sex twins $(\mathrm{OR}=0.23,95 \% \mathrm{CI}$ $0.18-0.28$ ), with overall rates of shared peers in women $88.0 \%$ in $\mathrm{MZ}$ twins, $69.8 \%$ in $\mathrm{DZ}$ same-sex twins and $49.2 \%$ in DZ unlike-sex twins, with corresponding percentages in men of $87.5 \%, 73.4 \%$ and $38.6 \%$. 
As shown in Table I, in like-sex twins, controlling for zygosity group differences, SSI was significantly more common in those who reported sharing the same peers at ages 6-13 and (although more weakly) in those who reported being in the same classes at school, but was less commonly reported by respondents with a lifetime history of major depression by the time of their interview assessment. SSI was associated, albeit weakly, with reduced probability of becoming a regular smoker, and, in regular smokers, with reduced probability of reporting DSM-IV nicotine dependence and heavy smoking (Table II). Adjusted odds ratios were no longer significant after controlling for zygosity, sex, major depression, same classes and same peers, but these estimates were almost unchanged in magnitude. We may speculate that the association between SSI and reduced rates of these smoking outcomes is occurring because a subset of individuals who were low on individuals characteristics associated with propensity to smoke were persuaded to smoke (or coerced) by their cotwin. This would be consistent with the observation that regular smoking was less common in

Table I. Individual Predictors of Simultaneous Initiation of Smoking with Cotwin (SSI) in Same-Sex Twins from Pairs Concordant for Ever Smoking: Adjusted Odds Ratios and 95\% Confidence Intervals Estimated under a Multivariate Logistic Model are Shown

\begin{tabular}{|c|c|c|}
\hline & Odds ratio & $95 \%$ Confidence interval \\
\hline Monozygotic female & 1.76 & $1.42-2.18$ \\
\hline Monozygotic male & 2.32 & $1.86-2.91$ \\
\hline Dizygotic female $^{a}$ & 1.00 & - \\
\hline Dizygotic male & 1.68 & $1.33-2.11$ \\
\hline DSM-IV major depression & 0.83 & $0.71-0.97$ \\
\hline Shared peers, ages $6-13$ & 1.80 & $1.48-2.19$ \\
\hline Same classes at school & 1.25 & $1.03-1.50$ \\
\hline
\end{tabular}

${ }^{\mathrm{a} C}$ Comparison group.
MZ than in DZ pairs concordant for ever smoking, with MZ twins more likely to report SSI.

\section{Genetic Analyses}

We found no evidence for genetic influences on the reporting of SSI: under the most parsimonious model, with parameters constrained across gender, variation in SSI reporting was accounted for by significant shared environmental influences $(40 \%, 95 \%$ CI 18 $52)$, non-significant genetic influences $(7 \%, 95 \%$ CI 0 34 ) and significant non-shared environmental influences $(53 \%, 95 \%$ CI $44-62 \%)$. Likewise, analyses of the reporting of sharing the same peers found significant shared environmental influences $(55 \%, 95 \%$ CI $42-60 \%)$, non-significant genetic influences $(0 \%, 95 \%$ CI 0-18\%), and significant non-shared environmental influences $(45 \%, 95 \%$ CI $37-50 \%)$. We cannot meaningfully refer to genetic influences on SSI or on sharing of peers, since twin pairs in reality must either be concordant for SSI or concordant for no-SSI, or for sharing or not sharing peers. However, finding no significant evidence for genetic influences on the reporting of SSI or the reporting of sharing peers simplifies the interpretation of later results, since it eliminates a possible source of genetic confounding.

Results of genetic analyses of regular smoking (in ever smokers), and of heavy smoking, DSM-IV nicotine dependence, DSM-IV nicotine withdrawal, and HSI (all conditioned on regular smoking) are shown in Table III. Shown in the two right-most columns of Table III are the Chi-Square tests of heterogeneity of genetic and environmental parameters, between SSI and non-SSI pairs, conducted separately for females and for males. Out of 10 significance tests, only a single test reaches statistical significance, that for regular smoking in female twins $(p<0.002)$. For the remaining smoking outcomes in Table III overall estimates of genetic and shared and non-shared environmental variance components, pooled across gender and across

Table II. Association between Simultaneous Initiation of Smoking with Cotwin (SSI) and Other Smoking Outcome Measures in Same-Sex Twins from Pairs Concordant for Ever Smoking: Unadjusted and Adjusted Multivariate Odds Ratios [and 95\% Confidence Intervals (CI)]

\begin{tabular}{lcc}
\hline Smoking outcome & Unadjusted odds ratio (95\% CI) & Adjusted $^{\mathrm{a}}$ odds ratio $(95 \% \mathrm{CI})^{-}$ \\
\hline Regular smoking & $0.83(0.72-0.96)$ & $0.88(0.76-1.02)$ \\
Heavy smoking & $0.82(0.68-0.98)$ & $0.86(0.71-1.04)$ \\
DSM-IV nicotine dependence & $0.77(0.64-0.93)$ & $0.83(0.69-1.00)$ \\
DSM-IV nicotine withdrawal & $0.85(0.71-1.02)$ & $0.89(0.74-1.07)$ \\
HSI & $0.83(0.66-1.05)$ & $0.88(0.69-1.11)$ \\
\hline
\end{tabular}

HSI: Heaviness of Smoking Index.

${ }^{a}$ Adjusted for zygosity, sex, DSM-IV major depression, shared peers, and same classes. 
Table III. Results of Genetic Analyses: Standardized Variance Component Estimates (and 95\% Confidence Intervals) for Regular Smoking and Measures of Nicotine Dependence in Adult Female and Male Australian Twins

\begin{tabular}{|c|c|c|c|c|c|}
\hline & \multicolumn{3}{|c|}{ Variance component estimates } & \multicolumn{2}{|c|}{$\begin{array}{l}\text { Test of Heterogeneity by } \\
\text { SSI }\left(\chi^{2}, \mathrm{df}=3\right)\end{array}$} \\
\hline & $a^{2}$ & $c^{2}$ & $e^{2}$ & Females & Males \\
\hline Heavy smoking ${ }^{\mathrm{a}, \mathrm{b}, \mathrm{c}}$ & $46(9-60)$ & $3(0-31)$ & $51(40-65)$ & 1.12 & 2.42 \\
\hline DSM-IV nicotine dependence ${ }^{\mathrm{a}, \mathrm{b}, \mathrm{c}}$ & $33(0-45)$ & $0(0-29)$ & $67(55-82)$ & 0.48 & 1.59 \\
\hline DSM-IV nicotine withdrawal ${ }^{\mathrm{a}, \mathrm{b}, \mathrm{c}}$ & $36(8-48)$ & $0(0-21)$ & $64(52-77)$ & 0.08 & 0.13 \\
\hline $\mathrm{HSI}^{\mathrm{a}, \mathrm{b}, \mathrm{c}}$ & $59(36-71)$ & $0(0-17)$ & $41(29-54)$ & 0.22 & 0.11 \\
\hline Regular smoking: ${ }^{\mathrm{d}}$ & & & & $15.52 *$ & 2.36 \\
\hline Concordant SSI females* & $92(25-97)$ & $0(0-65)$ & $8(3-16)$ & & \\
\hline Non-SSI females* & $35(7-68)$ & $29(0-53)$ & $36(25-47)$ & & \\
\hline All males ${ }^{c}$ & $85(78-91)$ & $0(0-5)$ & $15(9-22)$ & & \\
\hline
\end{tabular}

SSI: simultaneous smoking initiation; HSI: Heaviness of Smoking Index.

${ }^{a}$ Among twins pairs who report a lifetime history of regular smoking.

${ }^{\mathrm{b}}$ Parameter estimates equated in men and women.

${ }^{\mathrm{c}}$ Parameter estimates equated in concordant and non-SSI groups.

${ }^{\mathrm{d}}$ Among twin pairs who report they have experimented with cigarettes.

${ }^{*} p<0.01$.

SSI status, are reported (under the headings $a^{2}, c^{2}$ and $e^{2}$ ), since we did not find evidence for significant interactions of genetic or environmental effects with gender, nor with SSI versus non-SSI status.

For female like-sex pairs, we found significantly lower non-shared environmental variance for regular smoking in SSI pairs $(8 \%)$ than in non-SSI pairs (36\%: see lower half of Table III). Under the full model estimating separate genetic, shared environmental and non-shared environmental parameters for SSI versus non-SSI pairs, very high heritability was obtained for SSI pairs (92\%) versus more modest heritability for non-SSI pairs $(35 \%)$. However, we were able to constrain either the additive genetic variance $\left(\chi_{1}^{2}=0.90, p>0.10\right)$ or the shared environmental variance $\left(\chi_{1}^{2}=0.12, p>0.10\right)$, to be equal across SSI and non-SSI groups, but not both $\left(\chi_{1}^{2}=14.42, p<0.001\right)$. Thus while shared familial variance differed significantly across groups, there was insufficient power to determine whether this was due to heterogeneity of shared environmental or genetic parameters. Under a model allowing for shared environmental heterogeneity, estimates were: SSI $c^{2}=23 \%, 95 \%$ CI $11-35 \%$, non-SSI $c^{2}=0 \%, 95 \%$ CI $0-3 \%, a^{2}=69 \%, 95 \%$ CI $57-78$; under a model allowing for genetic heterogeneity, estimates were: SSI $a^{2}=63 \%, 95 \%$ CI $41-90 \%$, non-SSI $a^{2}=36 \%$, $95 \%$ CI $10-65 \%, c^{2}=29 \%, 95 \%$ CI $3-49$.

Estimated female like-sex twin pair polychoric correlations for regular smoking, as a function of SSI and sharing the same peers (S-Peers), are summarized in Table IV. Three conclusions may be drawn from this table: (i) there is a progressive increase in the magnitude of twin pair correlations, from pairs who report neither SSI (Non-SSI) nor sharing of peers (NS-Peers), to those who report sharing of peers but not SSI, and then to those who report both sharing of peers and SSI (MZ: 0.46, 0.73, and 0.92; DZ: 0.28, 0.49 , and 0.64 ); (ii) conditioning upon sharing of peers or SSI, twin pair correlations remain higher for MZ pairs than for DZ pairs, consistent with the hypothesis of genetic influences on regular smoking, although these differences do not reach statistical significance for one group, the pairs who do not share peers and do not report SSI; and (iii) a higher proportion of DZ pairs (34\%) than MZ pairs (14\%) are falling into this last category, so that any increased shared environmental variance associated with SSI or with sharing peers will inflate estimates of additive genetic variance for smoking initiation. Relatively few pairs who report SSI also report that they do not share peers, so that confidence intervals for correlations for this latter group are extremely broad. The progressive increments in twin pair correlations are roughly equal in $\mathrm{MZ}$ pairs and DZ pairs, whether we compare non-SSI pairs who do versus do not share peers (increase of 0.27 in $\mathrm{MZ}$ pairs versus 0.21 in $\mathrm{DZ}$ pairs), or compare pairs who share peers who do versus do not report SSI (increase of 0.19 in $\mathrm{MZ}$ pairs, 0.15 in DZ pairs), consistent with the hypothesis that SSI and sharing of peers are both associated with increased shared environmental contributions to twin pair resemblance. 
Table IV. Female Twin Pair Polychoric Correlations ( $\rho$ ) (and 95\% Confidence Intervals) for Regular Smoking, and their Heterogeneity by Zygosity, as a Function of Simultaneous Smoking Initiation (SSI) and Sharing of Peers

\begin{tabular}{|c|c|c|c|c|}
\hline & \multicolumn{4}{|c|}{ Shared peers } \\
\hline & & S-Peers & & NS-Peers \\
\hline $\begin{array}{l}S S I \\
\mathrm{MZ} \\
\mathrm{DZ} \\
\text { Heterogeneity of (MZ vs. DZ) }\end{array}$ & $\begin{array}{l}N=142 \\
N=55\end{array}$ & $\begin{array}{l}\rho=0.92(0.83-0.96)^{\mathrm{a}} \\
\rho=0.64(0.27-0.86)^{(\mathrm{d})} \\
\chi^{2}=8.13, p=0.004\end{array}$ & $\begin{array}{l}N=26 \\
N=13\end{array}$ & $\begin{array}{l}\rho=0.93(0.66-0.996)^{\mathrm{a}} \\
\rho=0.05(-0.73-0.91)^{(\mathrm{d})} \\
\chi^{2}=5.49, p=0.02\end{array}$ \\
\hline $\begin{array}{l}\text { Non-SSI } \\
\mathrm{MZ} \\
\mathrm{DZ} \\
\text { Heterogeneity of (MZ vs. DZ) }\end{array}$ & $\begin{array}{l}N=254 \\
N=178\end{array}$ & $\begin{array}{l}\rho=0.73(0.60-0.83)^{\mathrm{b}(\mathrm{c})} \\
\rho=0.49(0.28-0.66)^{\mathrm{e}(\mathrm{f})} \\
\chi^{2}=7.14, p=0.008\end{array}$ & $\begin{array}{l}N=71 \\
N=128\end{array}$ & $\begin{array}{l}\rho=0.46(0.11-0.75)^{(\mathrm{c})} \\
\rho=0.28(0.00-0.53)^{(\mathrm{f})} \\
\chi^{2}=1.41, p=0.24\end{array}$ \\
\hline
\end{tabular}

Within zygosity group, correlations with the same letter superscript do not differ significantly, or differ only at the trend level (given in parentheses: $p<0.10$ ).

Notes: Chi-square tests of heterogeneity of correlations within zygosity groups below.

(SSI, Non-SSI denotes simultaneous smoking initiation or its absence.

S-Peers denotes twin pair usually or always shared the same peers, NS-Peers denotes never, rarely or sometimes shared the same peers.

denotes pooling across S-Peers, NS-Peers in footnotes below).

${ }^{\mathrm{a}} \mathrm{MZ}$ (SSI, S-Peers) $=\mathrm{MZ}$ (SSI, NS-Peers): $\chi^{2}=0.57, p=0.90$.

${ }^{\mathrm{b}} \mathrm{MZ}$ (Non-SSI, S-Peers) $=\mathrm{MZ}$ (SSI, .): $\chi^{2}=8.35, p=0.004$.

${ }^{\mathrm{c}} \mathrm{MZ}$ (Non-SSI, NS-Peers) $=$ MZ (Non-SSI, S-Peers): $\chi^{2}=3.63, p=0.06$; MZ (Non-SSI, NS-Peers) $=$ MZ (SSI, .): $\chi^{2}=14.15, p<0.001$.

${ }^{\mathrm{d}} \mathrm{DZ}$ (SSI, S-Peers) $=\mathrm{DZ}$ (SSI, NS-Peers): $\chi^{2}=3.33, p=0.07$.

${ }^{\mathrm{e}} \mathrm{DZ}$ (Non-SSI, S-Peers) $=$ DZ (SSI, S-Peers): $\chi^{2}=3.86, p=0.049$.

${ }^{\mathrm{f}} \mathrm{DZ}$ (Non-SSI, NS-Peers) $=\mathrm{DZ}$ (Non-SSI, S-Peers): $\chi^{2}=3.71, p=0.054 ;$ DZ (Non-SSI, NS-Peers) $=\mathrm{DZ}$ (SSI, S-Peers): $\chi^{2}=4.57, p=0.03$.

A model that estimated three shared environmental parameters (baseline shared environmental variance in pairs reporting neither sharing of peers nor SSI; shared environmental variance increment in pairs reporting sharing of peers; shared environmental variance increment in pairs reporting SSI), as well as additive genetic and non-shared environmental effects, gave a good fit to the observed twin pair data $\left(\chi_{12}^{2}=8.32, p=0.76\right)$. Estimated variance components (and 95\% confidence intervals) were: non-shared environmental variance $7.7 \%(3-16 \%)$; additive genetic variance $56.1 \%(26-73 \%)$; baseline shared environmental variance $0 \%(0-28 \%)$; additional shared environmental variance associated with shared peers $16.6 \%(0-36 \%)$; additional shared environmental variance associated with SSI $19.6 \%$ $(8-32 \%)$. Because of the substantial overlap of sharing of peers and SSI, confidence intervals for the individual contributions of these terms are broad. However, under this model their combined effects account for $36 \%$ of the variance in risk of becoming a regular smoker (95\% CI $17-57 \%$ ). When we added to the model additional genetic variance associated with sharing of peers, this genetic parameter was estimated at its lower bound of zero. If instead we added to the model additional genetic variance associated with
SSI, there was a negligible improvement in the overall Chi-Square test of goodness-of-fit $\left(\chi_{1}^{2}=0.35\right.$, $p=0.55)$. Thus, the observed data are consistent with the hypothesis that there is significant additional environmental variance that is shared by twin pairs sharing the same peers, and by twin pairs reporting SSI, compared to non-SSI twins who do not share peers. When we ignored information about SSI and sharing of peers, fitting a standard ACE model, in contrast, estimated variance components were: nonshared environmental variance $22 \% \quad(16-30 \%)$; additive genetic variance $68 \%(38-84 \%)$; shared environmental variance $9 \%(0-37 \%)$. These results suggest that the unique social environmental features of twin pairs (high probability of sharing the same peers, and other environmental factors associated with SSI) are causing us to overestimate (albeit to a modest degree) the magnitude of genetic influences on regular smoking in women, and to underestimate the importance of shared environmental influences, unless these effects are explicitly modeled.

\section{DISCUSSION}

The goal of this study was to examine the extent to which early social environmental influences on 
smoking initiation (which may be more highly correlated in MZ than in DZ pairs) may lead to misleading inferences about genetic contributions to variation in later stages of smoking from twin data. We took advantage of the unique informativeness of twin pairs for assessing the persistence of effects of early social influences on later smoking outcomes, by comparing outcomes in twin pairs who did versus did not report simultaneous smoking initiation (SSI). We found no significant genetic influences on reporting of either SSI or sharing of peers, removing one possible source of genetic confounding in our analyses. SSI was influenced by gender (more likely in males, perhaps because of greater likelihood of opposite sex peer influences on smoking initiation in females), zygosity (more likely in MZ pairs), sharing the same peers, and being in the same classes at high school, in addition to being negatively associated with DSM-IV major depression. SSI was weakly associated with differences in later smoking outcomes, with twins reporting SSI being less likely to progress to regular smoking, and among regular smokers, being less likely to report heavy smoking and DSM-IV nicotine dependence. We may speculate that some twins who had low overall propensity to become smokers were induced to experiment with cigarettes by their cotwins (however, in additional analyses, we do not find, as we might anticipate from the higher proportion of SSI pairs in monozygotic pairs, a significantly lower rate of never smokers in MZ twins).

With the exception of regular smoking in females, estimates of genetic and shared and nonshared environmental variance components for smoking outcomes did not vary significantly between SSI versus non-SSI pairs. We can thus be reasonably confident that early social influences on initiation of smoking that may plausibly be hypothesized to be more highly correlated in MZ than in DZ pairs (e.g. shared peers) are not seriously biasing estimates of genetic effects on later stages of smoking. For regular smoking, for females only, we did find significant heterogeneity of twin pairs correlations as a function of both SSI and shared peers. It is possible that these influences are leading us to overestimate the importance of genetic influences on transition to regular smoking in women. However, we cannot exclude the possibility that we are dealing with a genotype-environment correlation effect, with heritable characteristics of twins leading to the selection of more similar peers and other more similar environments that make simultaneous smoking initiation more likely. Even under the more conservative assumption of excess environmental resemblance in $\mathrm{MZ}$ compared to $\mathrm{DZ}$ twin pairs associated with SSI and shared peers, adjusting for these effects only modestly reduced our overall heritability estimate for regular smoking in women ( $68 \%$ unadjusted versus $56 \%$ adjusted heritability). This finding gives more confidence in the usefulness of regular smoking as a phenotype for genetic research.

One potential confounding factor in our analyses is that of age at initiation of smoking, since twin pairs with later onset of smoking are less likely to report SSI. This factor may actually be causing us to underestimate the magnitude of SSI effects. For those individuals with age-of-initiation of smoking beyond the 75 th percentile (16 years and older), a lower proportion of individuals progressed to regular smoking $(49.7 \%$ vs. $58.8 \%)$, so this subset of non-SSI individuals will look more like SSI individuals in terms of their reduced likelihood of progression to regular smoking.

Several additional limitations to our findings need to be acknowledged. We excluded from our analysis twins who reported no history of cigarette use. To the extent that there is overlap of genetic influences on first use versus later smoking outcomes, this has the potential to bias estimates of genetic and environmental parameters (e.g. Heath et al., 2002). However, since $90 \%$ of our sample had used cigarettes at least once, this bias is likely to be rather minor. For analyses of smoking outcomes other than regular smoking, we intentionally limited consideration to pairs concordant for regular smoking, in our examination of SSI effects on heritability estimates. This will underestimate the true heritability of these phenotypes, because it excludes genetic variance associated with risk of becoming a regular smoker, and indeed leads to genetic variance estimates that are lower than those that we have reported elsewhere for the same sample (Lessov et al., 2004). A necessary future step will be to jointly analyze genetic effects on initiation of regular smoking and on smoking outcomes conditional upon regular smoking using models that have been developed for this purpose (e.g. Heath et al., 2002), taking into account factors such as SSI and sharing of peers which, if ignored, might be expected to cause such models to give a poor fit to the observed data. We only assessed limited aspects of early social influences pertinent to smoking initiation: for example, the study's measure of shared peers does not distinguish effects of peers' smoking. However, we would expect the totality of such early social influences on risk of smoking 
initiation to contribute to probability of SSI, so using SSI as a proxy measure should give us a good estimate of the persistence of effects of early social influences on later smoking outcomes. Finally, the behaviors in this study were assessed retrospectively in adult twins, and the extent to which these findings will be confirmed prospectively in adolescent and preadolescent samples needs to be pursued in future studies.

The scope of this study provides an important test of early social influences across a wide variety of early and later-stage smoking-related behaviors in both males and females, considering both smokingspecific (i.e. SSI) and more general experiential (i.e. shared peer) influences. It thus builds upon and extends earlier work (e.g. Kendler and Gardner, 1998; Madden et al., 2004; Slomkowski et al., 2005) suggesting that familial estimates of early, but not later, stages of smoking may be influenced by early environmental experiences. Our findings for regular smoking in female twins suggest the need for greater attention to the inclusion of such early social environment indicators in genetic modeling analyses. Our findings also suggest that the importance of early social influences for smoking initiation is unlikely to impede the successful application of genetic research strategies (whether twin study or genetic linkage or association) to later stages of smoking behaviors. These results should thus reduce a concern raised by some critics of genetic (or genomic) research on smoking (Merikangas and Risch, 2003).

\section{ACKNOWLEDGMENTS}

The authors are thankful for Support from the following NIH Grants: AA07728, AA11998, and AA13321 (A.C.H.), DA12540 and DA12854 (P.A.F.M), DA019951 and AA07535 (M.L.P), and the Australian National Health and Medical Research Council. The authors are also thankful to the twins and their families for participation in this research.

\section{REFERENCES}

American Psychiatric Association (1994). Diagnostic and Statistical Manual of Mental Disorders (Fourth Edition). Washington, DC: American Psychiatric Association.

Bucholz, K. K., Cadoret, R., and Cloninger, C. R., et al. (1994). A new semi-structured psychiatric interview for use in genetic linkage studies: a report of the reliability of the SSAGA. J. Studies Alcohol 55:149-158.
Center for Disease Control and Prevention (1998). Selected cigarettes smoking initiation and quitting behaviors among high school students-United States, 1997. Morb. Mortal. Wkly. 47(19):386-389.

Center for Disease Control and Prevention (2002). Annual smoking-attributable mortality, years of potential life lost, and economic costs-United States, 1995-1999. Morb. Mortal. Wkly. 51(14):300-302.

Center for Disease Control and Prevention (2004). Cigarette use among high school students-United States, 1991-2003. Morb. Mortal. Wkly. 53(23):499-502.

Center for Disease Control and Prevention (2005). Annual smoking-attributable mortality, years of potential life lost, and economic costs-United States, 1997-2001. Morb. Mortal. Wkly. 54(25):625-628.

Cleveland, H. H., Wiebe, R. P., and Rowe, D. C. (2005). Sources of exposure to smoking and drinking friends among adolescents: a behavioral-genetic evaluation. J. Genetic Psychol. 166(2):153169.

Cottler, L. B., Robins, L. N., and Grant, B. F., et al. (1991). The CIDI-core substance abuse and dependence questions: Crosscultural and nosological issues. The WHO/ADAMHA field trial. Br. J. Psychiatry 159:653-658.

Eaves, L. J., Last, K. A., Young, P. A., and Martin, N. G. (1978). Model-fitting approaches to the analysis of human behavior. Heredity 41:249-320.

Heath, A. C., Cates, R., Martin, N. G., Meyer, J., Hewitt, J. K., Neale, M. C., and Eaves, L. J. (1993). Genetic contribution to risk of smoking initiation: comparison across birth cohorts and across cultures. J. Subst. Abuse 5:221-246.

Heath, A. C., Howells, W., Kirk, K. M., Madden, P. A. F., Bucholz, K. K., Nelson, E. C., Slutske, W. S., Statham, D. J., and Martin, N. G. (2001). Predictors of non-response to a questionnaire survey of a volunteer twin panel: findings from the Australian 1989 twin cohort. Twin Res. 4(2):73-80.

Heath, A. C., and Madden, P. A. F. (1995). Genetic influences on smoking behaviors. In J. R. Turner, L. R. Cardon, and J. K. Hewitt (eds.), Behavior Genetic Approaches in Behavioral Medicine. New York: Plenum, pp. 45-66.

Heath, A. H., Martin, N. G., Lynskey, M. T., Todorov, A. A., and Madden, P. A. F. (2002). Estimating two-stage models for genetic influences on alcohol, tobacco or drug use initiation and dependence vulnerability in twin and family data. Twin Res. 5(2):113-124.

Heatherton, T. F., Kozlowski, L. T., Frecker, R. C., and Fagerstrom, K. O. (1991). The Fagerstrom test for nicotine dependence: a revision of the Fagerstrom Tolerance Questionnaire. Br. J. Addict. 86(9):1119-1127.

Heatherton, T. F., Kozlowski, L. T., Frecker, R. C., Rickert, W., and Robinson, J. (1989). Measuring the heaviness of smoking: using self-reported time to the first cigarette of the day and number of cigarettes smoked per day. Br. J. Addict. 84(7):791799.

Kendler, K. S., and Gardner, C. O. Jr. (1998). Twin studies of adult psychiatric and substance dependence disorders: are they biased by differences in the environmental experiences of monozygotic and dizygotic twins in childhood and adolescence? Psychol. Med. 28(3):625-633.

Kendler, K. S., Neale, M. C., Sullivan, P., Corey, L. A., Gardner, C. O., and Prescott, C. A. (1999). A population-based twin study in women of smoking initiation and nicotine dependence. Psychol. Med. 29:299-308.

Knopik, V. S., Heath, A. C., Madden, P. A. F., Bucholz, K. K., Slutske, W. S., Nelson, E. C., Statham, D., Whitfield, J. B., and Martin, N. G. (2004). Genetic effects on alcohol dependence risk: re-evaluating the importance of psychiatric and other heritable risk factors. Psychol. Med. 34(8):1519-1530.

Koopmans, J. R., Slutske, W. S., Heath, A. C., Neale, M. C., and Boomsma, D. I. (1999). The genetics of smoking initiation and 
quantity smoked in Dutch adolescent and young adult twins. Behav. Genet. 29(6):383-393.

Lessov, C. N., Martin, N. G., Statham, D. J., Todorov, A. A., Slutske, W. S., Bucholz, K. K., Heath, A. C., and Madden, P. A. F. (2004). Defining nicotine dependence for genetic research: evidence from Australian twins. Psychol. Med. 34:865-879.

Li, M. D., Cheng, R., Ma, J. Z., and Swan, G. E. (2003). A metaanalysis of estimated genetic and environmental effects on smoking behavior in male and female twins. Addiction 98:23-31.

Madden, P. A. F., Heath, A. C., Pedersen, N. L., Kaprio, J. K., Koskenvuo, M. J., and Martin, N. G. (1999). The genetics of smoking persistence in men and women: a multicultural study. Behav. Genet. 29:421-429.

Madden, P. A. F., Pedersen, N. L., Kaprio, J., Koskenvou, M. J., and Martin, N. G. (2004). The epidemiology and genetics of smoking initiation and persistence: crosscultural comparisons of twin study results. Twin Res. 7(1):82-97.

Maes, H. H., Sullivan, P. F., Bulik, C. M., Neale, M. C., Prescott, C. A., Eaves, L. J., and Kendler, K. S. (2004). A twin study of genetic and environmental influences on tobacco initiation, regular tobacco use and nicotine dependence. Psychol. Med. 34:1251-1261.

Merikangas, K. R., and Risch, N. (2003). Genomic priorities and public health. Science 302:599-601.

Neale, M. C., Boker, S. M., Xie, G., and Maes, H. H. (2002). $M X$. Statistical Modeling (6th ed.). Richmond, VA: Department of Psychiatry, Medical College of Virginia.

Peto, R., Darby, S., Deo, H., Silcocks, P., Whitley, E., and Doll, R. (2000). Smoking, smoking cessation, and lung cancer in the
UK since 1950: combination of national statistics with two case-control studies. Br. Med. J. 321:323-329.

Pomerleau, O. F., Collins, A. C., Shiffman, S., and Pomerleau, C. S. (1993). Why some people smoke and others do not: new perspectives. J. Consult. Clin. Psychol. 61:723-732.

SAS Institute Inc. (1999). SAS® Procedures Guide, Version 8. Cary, NC: SAS Institute Inc.

Simons-Morton, B. G. (2002). Prospective analysis of peer and parent influences on smoking initiation among early adolescents. Prev. Sci. 3(4):275-283.

Slomkowski, C., Rende, R., Novak, S., Lloyd-Richardson, E., and Niaura, R. (2005). Sibling effects on smoking in adolescence: evidence for social influence from a genetically informative design. Addiction 100(4):430-438.

StataCorp (2003). Stata statistical software, Release 8.0. College Station, TX: Stata Corporation.

Thomson, C. C., Fisher, L. B., Winickoff, J. P., Colditz, G. A., Camargo, C. A. Jr., King, C. III, and Frazier, A. L. (2004). State tobacco excise taxes and adolescent smoking behaviors in the United States. J. Public Health Manage. Practice 10(6):490-496.

Vink, J. M., Willemsen, G., and Boomsma, D. I. (2003). The association of current smoking behavior with the smoking behavior of parents, siblings, friends and spouses. Addiction 98(7):923-931. 